\title{
New Treatment of Strongly Anisotropic Scattering Phase Functions: The Delta-M+ Method
}

\author{
Zhenyi Lin, NAn Chen, YongZhen FAn, Wei Li, And Knut Stamnes \\ Department of Physics, Stevens Institute of Technology, Hoboken, New Jersey \\ SNORRE STAMNES \\ NASA Langley Research Center, Hampton, Virginia
}

(Manuscript received 10 August 2017, in final form 6 November 2017)

\begin{abstract}
The treatment of strongly anisotropic scattering phase functions is still a challenge for accurate radiance computations. The new delta- $M+$ method resolves this problem by introducing a reliable, fast, accurate, and easy-to-use Legendre expansion of the scattering phase function with modified moments. Delta- $M+$ is an upgrade of the widely used delta- $M$ method that truncates the forward scattering peak with a Dirac delta function, where the "+" symbol indicates that it essentially matches moments beyond the first $M$ terms. Compared with the original delta- $M$ method, delta- $M+$ has the same computational efficiency, but for radiance computations, the accuracy and stability have been increased dramatically.
\end{abstract}

\section{Introduction}

In most current scalar radiative transfer models for radiance computations in plane-parallel geometry (Stamnes et al. 1988, 2000; Jin et al. 2006; Spurr 2008; Rozanov et al. 2014; Lin et al. 2015; Stamnes and Stamnes 2016; Hamre et al. 2017; K. Stamnes et al. 2017), the scattering phase function is expanded in a finite series of Legendre polynomials. This expansion of the scattering phase function combined with an expansion of the radiance in a Fourier cosine series leads to a radiative transfer equation (RTE) for each Fourier component that is azimuth independent and mathematically identical for all Fourier components (Stamnes et al. 1988). As a result, an accurate and stable RTE solution relies on an adequate expansion of the scattering phase function, which could be computationally expensive, because a strongly asymmetric scattering phase function may require hundreds of terms in a standard Legendre polynomial expansion. For example, Kokhanovsky et al. (2010) used 480 terms to expand a scattering phase function for a polydispersion of aerosol particles and 720 terms for a polydispersion of (water) cloud droplets in order to produce accurate radiative transfer benchmark results.

To alleviate the computational burden, truncation methods (Joseph et al. 1976; Wiscombe 1977; Nakajima

Corresponding author: Zhenyi Lin, lzhenyi@stevens.edu and Tanaka 1988; Hu et al. 2000; Mitrescu and Stephens 2004) have been introduced in which the required number of terms in the expansion is reduced by replacing the sharp forward scattering peak by a Dirac delta function. Two popular truncation methods are the $\delta$ - $M$ (Wiscombe 1977) and the $\delta$-fit (Hu et al. 2000) methods. Overall, combined with the single-scattering correction (Nakajima and Tanaka 1988), the $\delta$ - $M$ method, in comparison with other truncations methods, was shown to provide the most accurate radiances for scattering phase functions that are not too strongly forward peaked (Rozanov and Lyapustin 2010). The $\delta$ - $M$ algorithm is implemented in many radiative transfer models including DISORT (Stamnes et al. 1988) used in MODTRAN (Berk et al. 2014).

Nevertheless, none of the current truncation methods is perfect (Rozanov and Lyapustin 2010). The $\delta$ - $M$ method, designed for irradiance calculations, was found to be unable to provide accurate radiances for strongly anisotropic scattering. The $\delta$-fit method can provide accurate radiances (except in the forward direction) but requires an ad hoc specification of the truncation angle and a higher computational burden than $\delta$ - $M$. A $\delta$-fit user must specify by trial and error the "best" truncation angle for each scattering phase function, and the least squares fitting employed in the $\delta$-fit method also implies additional computations. These problems make the $\delta$-fit method inconvenient to use and slower than the $\delta$ - $M$ method. 
In this paper, we will address these issues by introducing a new truncation technique, the $\delta-M+$ method, designed for efficient yet accurate computation of radiances in turbid media with strongly asymmetric scattering phase functions. The new $\delta-M+$ method represents an extension and upgrade of the standard $\delta$ - $M$ algorithm, which leads to a significant improvement in accuracy, while retaining the same computational efficiency and "user friendliness" as the original $\delta$ - $M$ method (Wiscombe 1977).

\section{Review of the delta- $M$ method}

In a full-range slab geometry, the radiative transfer equation for the diffuse radiance may be written as (K. Stamnes et al. 2017)

$$
u \frac{d I(\tau, u, \phi)}{d \tau}=I(\tau, u, \phi)-\frac{\varpi}{4 \pi} \int_{0}^{2 \pi} d \phi^{\prime} \int_{-1}^{1} d u^{\prime} p\left(u^{\prime}, \phi^{\prime} ; u, \phi\right) I\left(\tau, u^{\prime}, \phi^{\prime}\right)-(1-\varpi) B-S^{*}(\tau, u, \phi),
$$

where $\tau$ is the optical depth, $u$ is the cosine of the polar angle $\theta, \phi$ is the relative azimuth angle, $\varpi$ is the single-scattering albedo, and $p\left(u^{\prime}, \phi^{\prime} ; u, \phi\right)$ is the scattering phase function, which describes the probability of light scattering from the incident direction $\left(u^{\prime}, \phi^{\prime}\right)$ to the scattered direction $(u, \phi)$. The single-scattering source term $S^{*}(\tau, u, \phi)$, proportional to the incident solar beam irradiance $F^{s}$, is given by

$$
S^{*}(\tau, u, \phi)=\frac{\varpi}{4 \pi} p\left(u^{\prime}, \phi^{\prime} ; u, \phi\right) F^{s} e^{-\tau / \mu_{0}},
$$

where $\mu_{0}$ is the cosine of the incident (solar) zenith angle. To obtain the radiance, we have to solve for a function of three variables, $\tau, u$, and $\phi$. But this three-variable problem can be reduced to one of solving a finite set of uncoupled radiative transfer equations, each depending on only the two variables $\tau$ and $u$. We start by expanding the scattering phase function in a finite series of $M$ Legendre polynomials as follows $\left[\cos \Theta=u u^{\prime}+\sqrt{1-u^{2}} \sqrt{1-u^{\prime 2}} \cos \left(\phi-\phi^{\prime}\right)\right]$ :

$$
\begin{aligned}
p(\cos \Theta) & =p\left(u^{\prime}, \phi^{\prime} ; u, \phi\right) \\
& \approx \sum_{\ell=0}^{M-1}(2 \ell+1) \chi_{\ell} P_{\ell}(\cos \Theta),
\end{aligned}
$$

where $\Theta$ is the scattering angle, $P_{\ell}$ is the $\ell$ th Legendre polynomial, and the $\ell$ th expansion coefficient is given by

$$
\chi_{\ell}=\frac{1}{2} \int_{-1}^{1} d(\cos \Theta) P_{\ell}(\cos \Theta) p(\cos \Theta) .
$$

To simplify the expansion of the scattering phase function we use the addition theorem for spherical harmonics (Arfken and Weber 1995):

$$
\begin{aligned}
P_{\ell}(\cos \Theta)= & P_{\ell}\left(u^{\prime}\right) P_{\ell}(u) \\
& +2 \sum_{m=1}^{\ell} \Lambda_{\ell}^{m}\left(u^{\prime}\right) \Lambda_{\ell}^{m}(u) \cos m\left(\phi^{\prime}-\phi\right),
\end{aligned}
$$

where we have introduced the normalized associated Legendre polynomial defined by

$$
\Lambda_{\ell}^{m}(u) \equiv \sqrt{\frac{(\ell-m) !}{(\ell+m) !}} P_{\ell}^{m}(u),
$$

where $P_{\ell}^{m}(u)$ is the associated Legendre polynomial.

In the $\delta$ - $M$ method, we introduce an approximate scattering phase function $p^{*}(\cos \Theta)$ (Wiscombe 1977):

$$
\begin{aligned}
p^{*}(\cos \Theta)= & 2 f \delta(1-\cos \Theta) \\
& +(1-f) \sum_{\ell=0}^{M-1}(2 \ell+1) \chi_{\ell}^{*} P_{\ell}(\cos \Theta),
\end{aligned}
$$

where the first term is the Dirac $\delta$ function and $f$ is the truncation factor. The second term on the right is called the "truncated scattering phase function," whose coefficients $\chi_{\ell}^{*}$ are determined by matching them to the $\chi_{\ell}$ of the accurate (true) scattering phase function, given by Eq. (4). This requirement leads to

$$
\begin{gathered}
\chi_{\ell}^{*}=\frac{\chi_{\ell}-f}{1-f}, \quad \ell=0, \ldots, M-1 \text { and } \\
f=\chi_{M} .
\end{gathered}
$$

After the truncation, the radiative transfer equation is unchanged except that the optical depth $\tau$ and the singlescattering albedo $\varpi$ are scaled as follows:

$$
\begin{aligned}
d \tau^{\prime} & =(1-\varpi f) d \tau \quad \text { and } \\
\varpi^{\prime} & =\frac{1-f}{1-\varpi f} \varpi,
\end{aligned}
$$

where $\tau^{\prime}$ and $\varpi^{\prime}$ are the scaled variables resulting from using the approximate scattering phase function in Eq. (7).

\section{The error of the delta- $M$ truncation}

Because of the orthogonality of the Legendre polynomials, the Dirac delta function in Eq. (7) may be expanded in an infinite series of Legendre polynomials: 

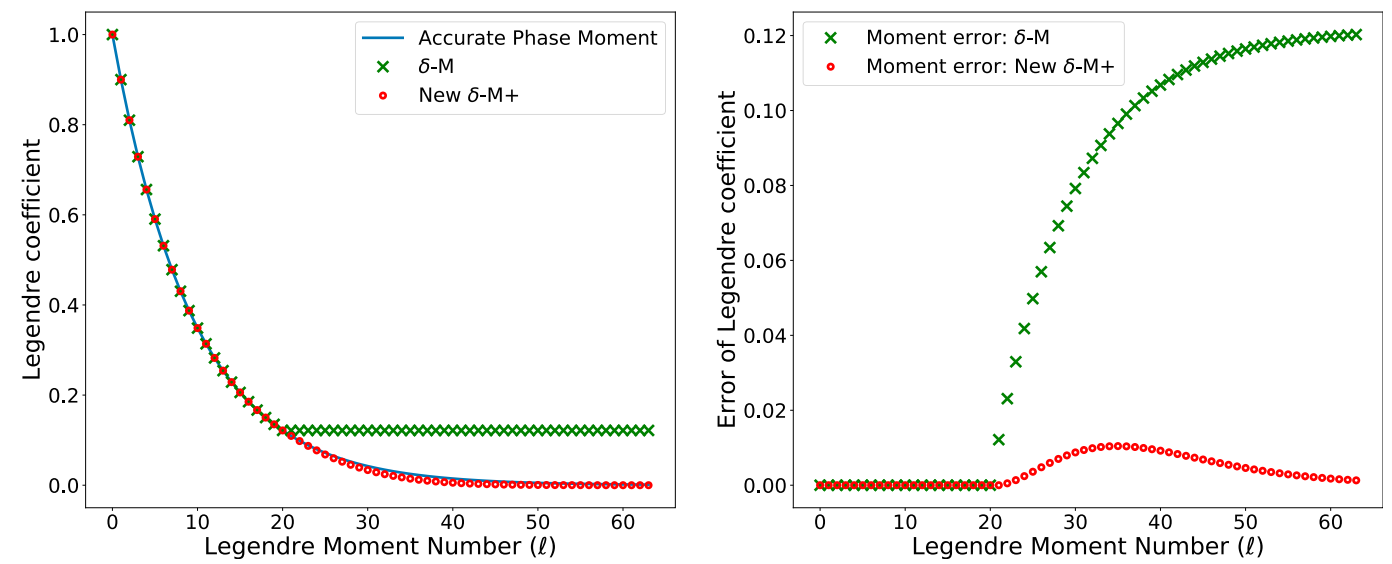

FIG. 1. (left) Moments and (right) moment errors of a 20-term $\delta$ - $M$ and $\delta$ - $M+$ representation of an HG scattering phase function with asymmetry factor $g=0.9$.

$$
2 f \delta(1-\cos \Theta)=\sum_{\ell=0}^{\infty}(2 \ell+1) f P_{\ell}(\cos \Theta) .
$$

In Eq. (12), a very important feature is the constant coefficient $f$ for all terms in the sum. Substituting Eqs. (8) and (12) into Eq. (7), we find that the approximate scattering phase function becomes

$$
\begin{aligned}
p^{*}(\cos \Theta)= & \sum_{\ell=0}^{M-1}(2 \ell+1) \chi_{\ell} P_{\ell}(\cos \Theta) \\
& +\sum_{\ell=M}^{\infty}(2 \ell+1) f P_{\ell}(\cos \Theta) .
\end{aligned}
$$

We note that the first $M$ terms in Eq. (13) contain the standard Legendre expansion coefficients (or moments) $\chi_{\ell}$ given by Eq. (4). Hence, the error incurred by applying the approximate scattering phase function is included in the higher-order terms with $\ell \geq M$ :

$$
\begin{aligned}
\varepsilon & =p(\cos \Theta)-p^{*}(\cos \Theta) \\
& =\sum_{\ell=M}^{\infty}(2 \ell+1)\left(\chi_{\ell}-f\right) P_{\ell}(\cos \Theta) .
\end{aligned}
$$

Figure 1 shows an example of $\delta-M$ and $\delta-M+$ moments (Legendre polynomial expansion coefficients) for a Henyey-Greenstein (HG) scattering phase function approximated by a truncated 20-term $(M=20)$ expansion. We note that the first 20 moments are accurate, but the error for higher-order moments $(\ell \geq 20$, shown as $\left.\chi_{\ell}^{*}-\chi_{\ell}\right)$ is much larger in the $\delta$ - $M$ approximation than in the $\delta-M+$ approximation.

\section{A modified representation of the Dirac delta function}

The $\delta$ - $M$ method, which assumes a constant truncation for all Legendre moments, leads to large errors in higher-order moments. To overcome this problem, we introduce a weighted Legendre series for the delta function as a new approximation of the Dirac delta function $\delta^{*}(1-\cos \Theta)$, which we write as

$$
\begin{aligned}
2 f \delta^{*}(1-\cos \Theta) & =\sum_{\ell=0}^{\infty}(2 \ell+1) w_{\ell} f P_{\ell}(\cos \Theta) \quad \text { and } \\
w_{\ell} & =\operatorname{cexp}\left(-\frac{\ell^{2}}{2 \sigma^{2}}\right), \quad \ell=0,1,2, \ldots
\end{aligned}
$$

In Eq. (15), the coefficients are changed from a constant value $f$ to the weighted values $w_{\ell} f$. The purpose of introducing weights is to produce a new function that is still sharply peaked and looks close to a delta function but also, by a suitable choice of the weights, agrees better with the actual scattering phase function for higher-order moments $(\ell>M)$. For simplicity, we assumed that the weights $w_{\ell}$ have a Gaussian distribution as shown in Eq. (16), where the value of $c$ and $\sigma$ are to be determined by matching $2 \delta^{*}(1-\cos \Theta)$ to the higher order of Legendre moments described in the next section. Applying the approximate $\delta$ function given by Eq. (15), we now introduce a new, improved approximate scattering phase function, given by

$$
\begin{aligned}
p_{\text {new }}^{*}(\cos \Theta)= & 2 f \delta^{*}(1-\cos \Theta) \\
& +\sum_{\ell=0}^{M-1}(2 \ell+1)\left(\chi_{\ell}-w_{\ell} f\right) P_{\ell}(\cos \Theta) .
\end{aligned}
$$

Figure 1 shows how moment errors $\left(\chi_{\ell}^{*}-\chi_{\ell}\right)$ in higher orders are reduced after using the new approximate scattering phase function (which is labeled in the figure as "New $\delta$ - $M+$ ").

One may ask what the new approximate function $\delta^{*}(1-\cos \Theta)$ would look like. Figure 2 shows an example of the shape of $w_{\ell}$ and the corresponding $\delta^{*}(1-\cos \Theta)$ for 

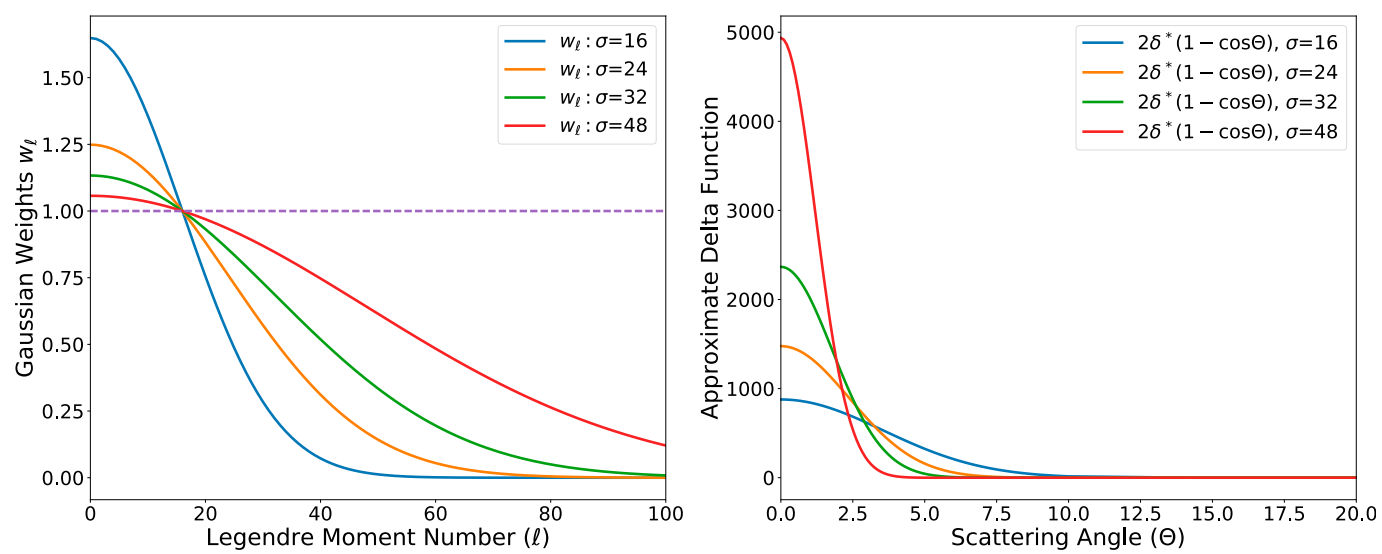

FIG. 2. (left) Gaussian weights and (right) corresponding new approximate delta function $\delta^{*}(1-\cos \Theta)$, where $c$ is determined by normalizing $w_{16}=1$. The horizontal dashed line corresponds to the condition without weighting $\left(w_{\ell}=1\right)$.

different combinations of $c$ and $\sigma$. Looking at Fig. 2, we first realize that the new approximate function is normalized to $w_{0}$, which is greater than 1.0. Therefore, it magnifies the truncation factor $f$ in the original $\delta$ - $M$ method. This magnification is desired because a larger forward-peak truncation is needed to truncate the strongly anisotropic scattering phase function. Second, the shape of the new function just looks like a sharp spike in the forward direction, whose magnitude and width are determined by the parameters $\sigma$ and $c$. These two parameters give us more freedom to control the sharp forward scattering peak. Third, as $\sigma$ increases, the lower-order moments of $w_{\ell}(\ell=0,1, \ldots, M-1)$ become closer to 1 , and we see that the new approximate delta function approaches the original delta function (e.g., for $\sigma=48$ in Fig. 2, $w_{\ell} \approx 1$, for $\ell=0,1$, $2, \ldots, 16)$. Under this condition, the $\delta$ - $M+$ truncation will approach the original $\delta-M$ truncation.

\section{The new $\delta-M+$ method}

Based on the new approximate Dirac delta function [Eq. (15)], we upgraded $\delta$ - $M$ and developed a new $\delta$ - $M+$ truncation method. Since in Wiscombe (1977), $M$ is essentially the order of the approximation $(M=1$ leads to the delta-two-stream or delta-Eddington approximation), we use the "+" sign to indicate an improved approximation for moments beyond the $M$ th moment by adjusting the values of the higher-order weights.

In general, the ideal way to match higher-order moments is to make every higher-order term of Legendre moments correct:

$$
w_{\ell} f=\chi_{\ell}, \quad \ell=M, M+1, M+2, \ldots
$$

However, since it is more important to get correct lowerorder moments than higher-order ones, and since we only have two Gaussian parameters ( $c$ and $\sigma$ ) available to control the shape of $\delta^{*}(1-\cos \Theta)$, we simply match the $M$ th and $(M+1)$ th Legendre moments by setting

$$
\left\{\begin{aligned}
w_{M} f & =\chi_{M} \\
w_{M+1} f & =\chi_{M+1}
\end{aligned}\right.
$$

Note that we also define $f=\chi_{M}$ as in the original $\delta$-M method so that $w_{M}=1$. The solution of Eq. (19) is straightforward and given by

$$
\begin{aligned}
f & =\chi_{M}, \\
w_{M} & =1, \\
\sigma & =\sqrt{\frac{(M+1)^{2}-M^{2}}{\ln \chi_{M}^{2}-\ln \chi_{M+1}^{2}}}, \text { and } \\
c & =w_{0}=\exp \left(\frac{M^{2}}{2 \sigma^{2}}\right) .
\end{aligned}
$$

The constant $c$ is determined by the normalization $w_{M}=$ 1 , and $c=w_{0}>1$, which could be considered as a magnification factor of $f$. Next, the truncated scattering phase function in Eq. (17) is normalized by dividing by $\left(1-w_{0} f\right)=\left(1-f^{\prime}\right)$ :

$$
\begin{aligned}
\chi_{\ell}^{*} & =\frac{\chi_{\ell}-w_{\ell} f}{1-f^{\prime}} \\
& =\frac{\chi_{\ell}-f^{\prime} \exp \left(-\ell^{2} / 2 \sigma^{2}\right)}{1-f^{\prime}}, \\
\ell & =0,1,2, \ldots, M-1, \quad \text { and } \\
f^{\prime} & =w_{0} f=\exp \left(M^{2} / 2 \sigma^{2}\right) \chi_{M},
\end{aligned}
$$

where we see that the new truncation factor $f^{\prime} \geq f$.

At last, we need to scale the optical depth $\tau$ and the single-scattering albedo $\varpi$ for the new method in a similar manner as in the $\delta$ - $M$ method: 

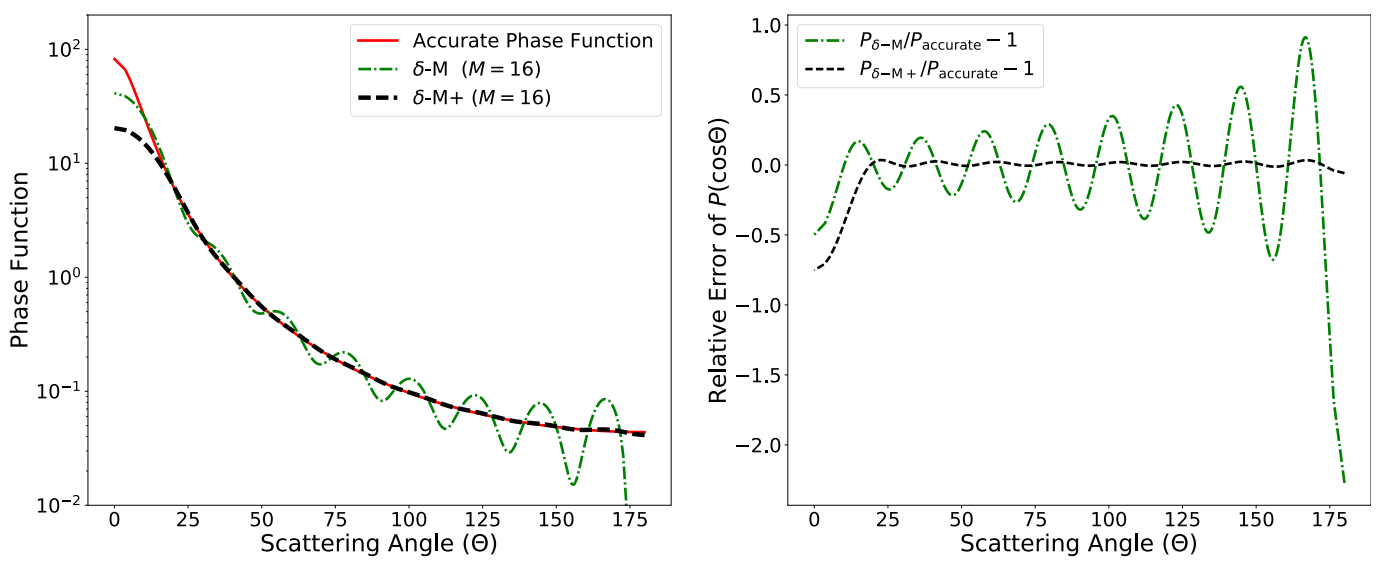

FIG. 3. (left) HG scattering phase function $(g=0.85)$. (right) Relative error incurred by expanding the HG scattering phase function. Sixteen terms are used for the Legendre polynomial expansion of the $\delta$ - $M$ and the new $\delta$ - $M+$ method. The magnitude of the parameters $\sigma$ and $c$ are listed in Table 1 .

$$
\begin{aligned}
& d \tau^{\prime}=\left(1-\varpi f^{\prime}\right) d \tau \text { and } \\
& \varpi^{\prime}=\frac{1-f^{\prime}}{1-\varpi f^{\prime}} \varpi .
\end{aligned}
$$

\section{Examples and comparison of scattering phase functions}

\section{a. Henyey-Greenstein scattering phase function}

The Legendre polynomial expansion of the HG scattering phase function (Henyey and Greenstein 1941) with asymmetry factor $g$ is

$$
p_{\mathrm{HG}}(\cos \Theta) \approx \sum_{\ell=0}^{M-1}(2 \ell+1) g^{\ell} P_{\ell}(\cos \Theta) .
$$

In Fig. 3, three HG scattering phase functions with asymmetry factor $g=0.85$ are shown in the left panel: the accurate scattering phase function, $\delta$ - $M$ truncated scattering phase function, and the new $\delta-M+$ truncated scattering phase function. Sixteen terms were used to expand the truncated scattering phase function. The right panel shows relative errors of the $\delta-M$ and the new $\delta-M+$ scattering phase functions.

Except for unavoidable errors around the forward peak, the new $\delta-M+$ scattering phase function looks essentially the same as the actual (true) scattering phase function at other angles, whereas the original $\delta$-M scattering phase function fluctuates around the true scattering phase function, whose relative error increases with scattering angle $\Theta$.

\section{b. Aerosol scattering phase function}

To test the new $\delta-M+$ method for large aerosol particles, we used the scattering phase function adopted in
Kokhanovsky et al. (2010)'s benchmark paper, where a Mie code was used to compute the scattering phase function at $412 \mathrm{~nm}$ assuming that the radius $a$ of the aerosol particles follows a lognormal distribution:

$$
f(a)=\frac{1}{\sqrt{2 \pi} s a} \exp \left[-\ln ^{2}\left(a / a_{0}\right) / 2 s^{2}\right],
$$

with $a_{0}=0.2 \mu \mathrm{m}, s=0.92$, and $a_{\text {end }}=30 \mu \mathrm{m}$. The value of $a_{\text {end }}$ specifies the upper limit of integration of Mie optical cross section with respect to the particle radius $a$. The lower limit of integration was equal to $0.005 \mu \mathrm{m}$. The refractive index of the aerosol particles was set to 1.385 .

Figure 4 shows the true, the $\delta$ - $M$, and the new $\delta-M+$ scattering phase functions for this aerosol case. The truncated scattering phase function is expanded using 32 terms. While the $\delta$ - $M$ method has a relative error up to $0.2(20 \%)$, the error has been greatly reduced for the new $\delta$ - $M+$ method.

\section{c. Water cloud scattering phase function}

Similar to the aerosol test, the cloud droplet distribution from Kokhanovsky et al. (2010)'s benchmark is used for the cloud case. As in the aerosol case, the

TABLE 1. Magnitude of the $\sigma$ and $c$ parameters of the $\delta$ - $M+$ in Figs. 3-8.

\begin{tabular}{lrc}
\hline \hline Figure No. & \multicolumn{1}{c}{$\sigma$} & $c$ \\
\hline 3 & 10.076 & 3.528 \\
4 & 31.700 & 1.664 \\
5 & 80.903 & 1.367 \\
6 & 180.232 & 1.016 \\
7 & 51.749 & 1.211 \\
$8(100$ terms $)$ & 162.532 & 1.208 \\
$8(200$ terms $)$ & 217.710 & 1.525 \\
\hline
\end{tabular}



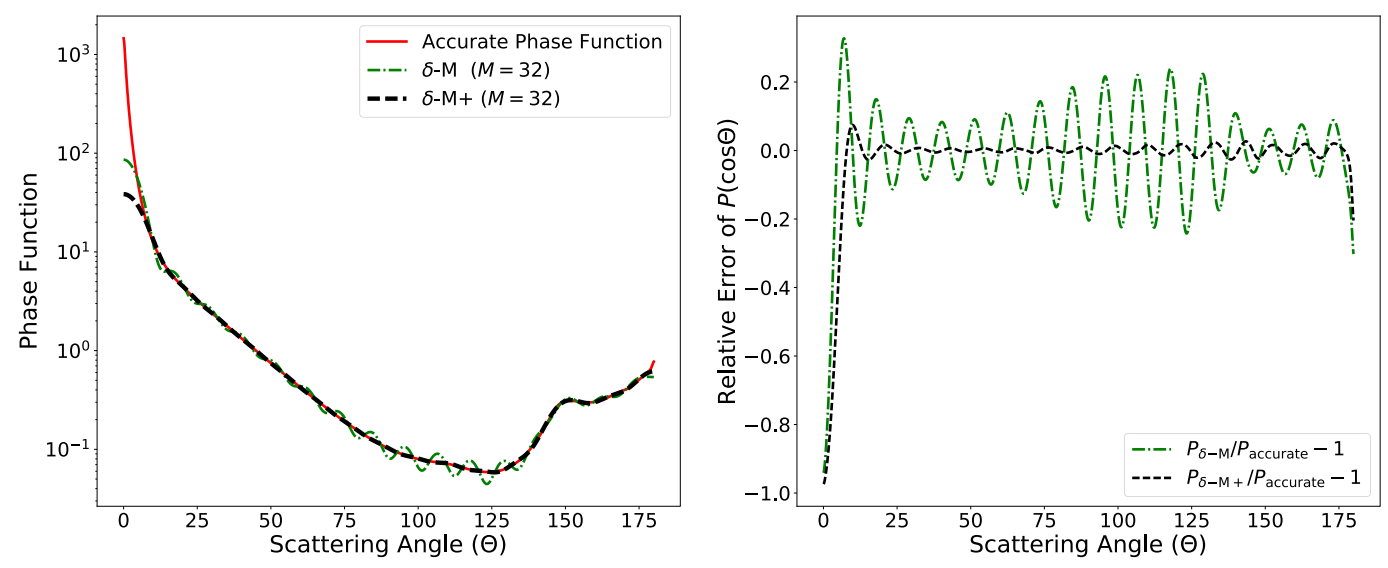

FIG. 4. (left) Kokhanovsky et al.'s (2010) aerosol scattering phase function. (right) Relative error incurred by expanding the aerosol scattering phase function. Thirty-two terms are used for the Legendre polynomial expansion of the $\delta$ - $M$ and new $\delta$ - $M+$ methods. The magnitude of the parameters $\sigma$ and $c$ are listed in Table 1 .

scattering phase function was computed by a Mie code based on a narrow lognormal distribution, where $a_{0}=5 \mu \mathrm{m}, s=0.4, a_{\mathrm{end}}=100 \mu \mathrm{m}$, and the refractive index was set to $m_{r}=1.339$. The maximum forward peak has a very large value reaching 7830, and there are pronounced rainbow and glory features (two more peaks). We used 64 terms to expand the truncated scattering phase function. Again, a comparison of relative errors shows that the new $\delta$ - $M+$ method greatly outperforms the old method and gives results with high accuracy (see Fig. 5).

\section{d. Stability test for extreme scattering phase functions}

We have already tested the $\delta$ - $M+$ method on large aerosol and water cloud particles and reduced the relative errors significantly, but we still do not know how well the $\delta-M+$ method automatically truncates extreme anisotropic scattering phase functions.

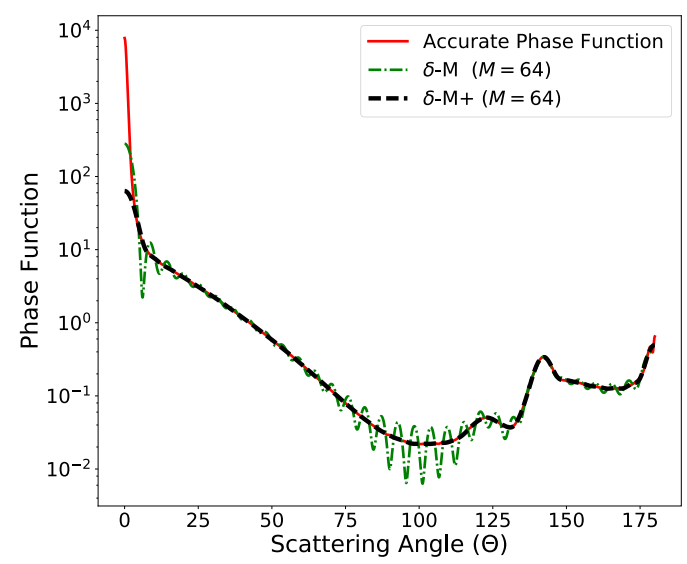

Two extreme scattering phase functions were introduced here based on the HG scattering phase function and the Fournier-Forand (FF) scattering phase function (Fournier and Forand 1994). In the first test, we used the HG scattering phase function with $g=0.999$, yielding a scattering phase function that is extremely forward peaked, whose peak magnitude reaches around 2000000 . We used only 32 terms to expand the truncated scattering phase function. The results show that new $\delta$ - $M+$ method works very well, while the original $\delta$ - $M$ method oscillates strongly and gives negative values (see Fig. 6).

Another sharply peaked scattering phase function is the FF function. We adopted a refractive index (real part, $m_{r}=1.0686$ ) typical of pigmented (phytoplankton) particles and used 32 terms to test the new $\delta$ - $M+$ method. Again, the new method was found to work well, while the original $\delta$ - $M$ method failed to converge as shown in Fig. 7.

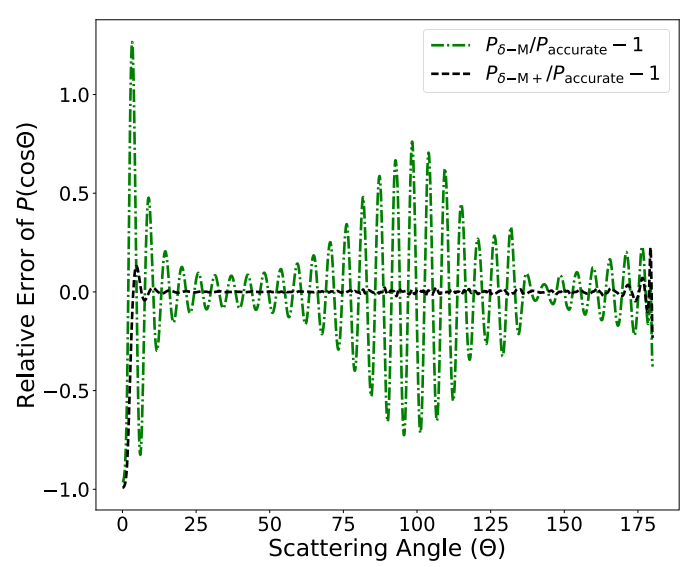

FIG. 5. As in Fig. 4, but for cloud scattering phase function with 64 terms. 

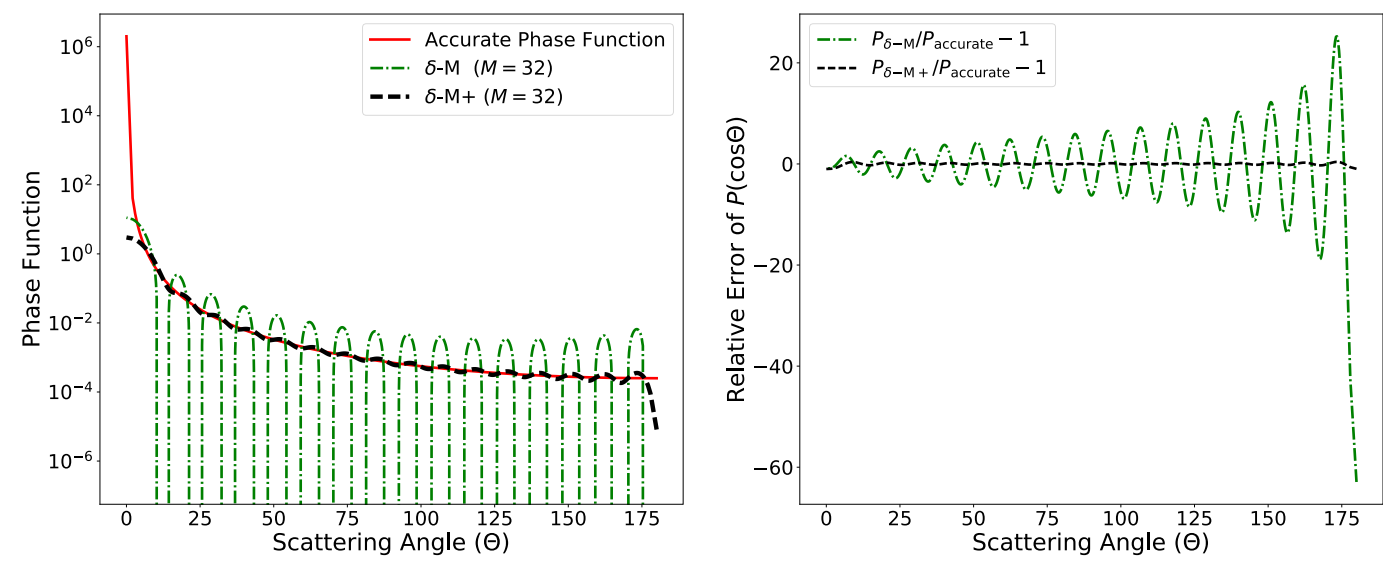

FIG. 6. As in Fig. 3, but for $g=0.999$ with 32 terms.

\section{e. Ice cloud scattering phase function}

Finally, we tested the expansion of an ice cloud scattering phase function at $0.55 \mu \mathrm{m}$ (Takano and Liou 1989). Compared with the water cloud scattering phase function, the scattering phase function of ice crystals is more challenging because it has not only a stronger forward peak (reaching 100000) but also halo peaks at other scattering angles that may not necessarily benefit from the truncation of the forward scattering peak. As a result, hundreds of expansion terms are required for accurately expanding those additional peaks even by the $\delta$ - $M+$ method. Applied 100 and 200 expansion terms, we obtained the results shown in Fig. 8. We see that the $\delta$ - $M+$ method has a better truncation of the forward peak than the original $\delta$ - $M$ method, which fails to adequately represent the ice crystal scattering phase function even when using 200 terms.

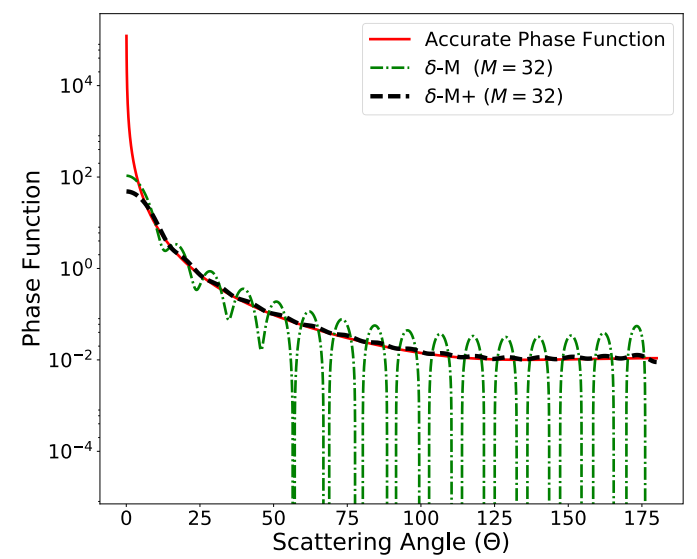

\section{Radiance tests}

From sections $6 \mathrm{~b}$ and $6 \mathrm{c}$, it is clear that the new $\delta$ - $M+$ method works well and provides accurate representations of the Kokhanovsky et al. (2010) aerosol and cloud scattering phase functions. Here, we first revisit these benchmarks to look at the relative error (\%) of the reflectance as a function of viewing polar angle. The Kokhanovsky benchmarks assume a homogeneous aerosol/ cloud slab and a direct beam incident at $60^{\circ}$ at the top of the slab. The optical depth of the slab was 0.3262 for the aerosol case and 5.0 for the cloud case, respectively.

In Fig. 9, relative errors of the Kokhanovsky et al. (2010) aerosol benchmark results are shown for the original $\delta$ - $M$ and the new $\delta$ - $M+$ methods. Generally, we see that the errors have been greatly reduced from $10 \%$ to less than $1 \%$ for most viewing angles. An exception happens around the exact backward scattering angle $\left(\Delta \phi=180^{\circ}, \theta=60^{\circ}\right)$. Considering that the reflectance is

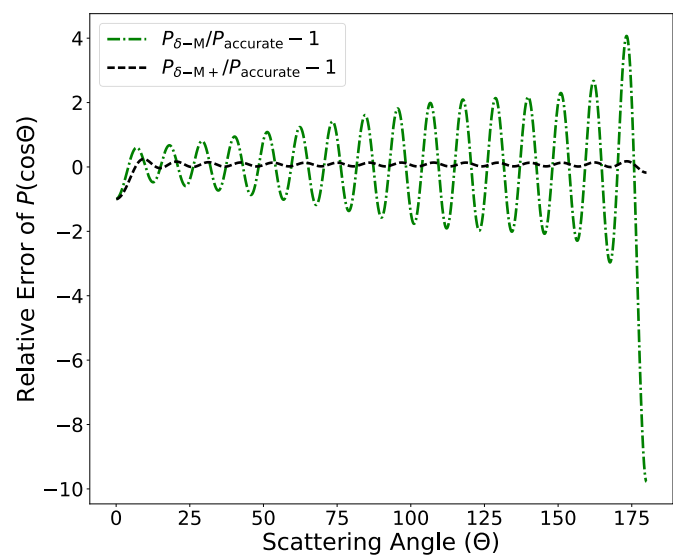

FIG. 7. (left) FF scattering phase function $\left(m_{r}=1.0686\right)$. (right) Relative error incurred by expanding the FF scattering phase function. Thirty-two terms are used for the Legendre polynomial expansion of the $\delta$ - $M$ and the new $\delta$ - $M+$ methods. The magnitude of the parameters $\sigma$ and $c$ are listed in Table 1. 

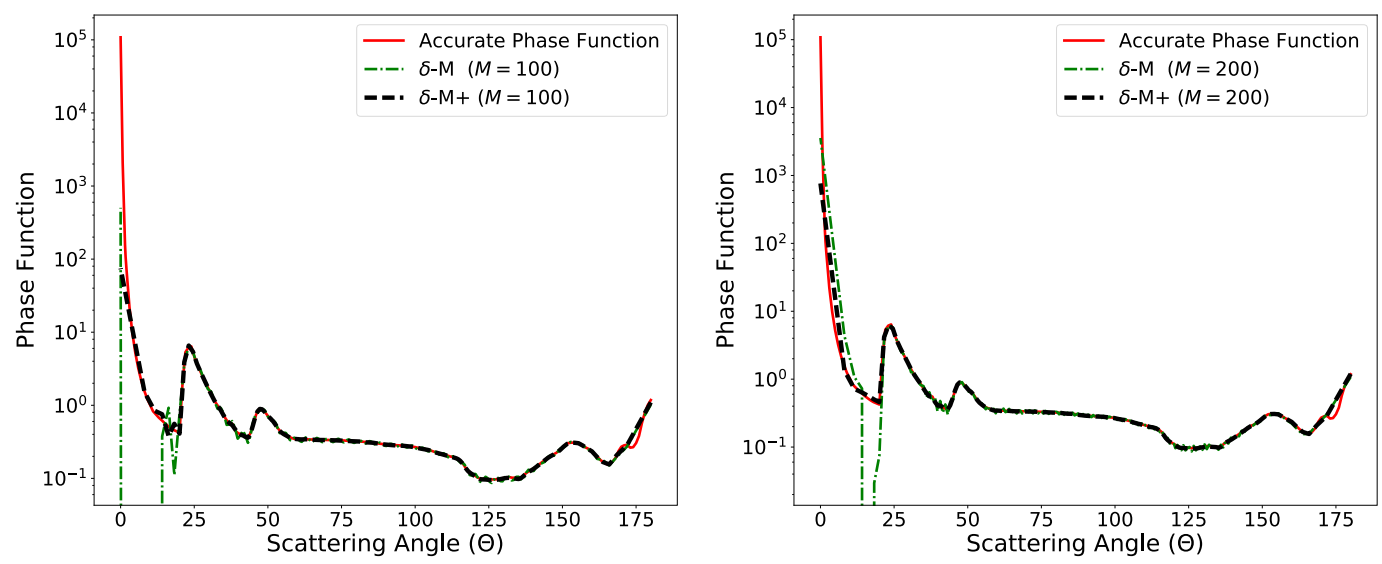

FIG. 8. Ice cloud scattering phase function expanded by (left) 100 and (right) 200 terms. The magnitude of the parameters $\sigma$ and $c$ are listed in Table 1.

very small at the backscattering angle and that we used only 32 streams to compute the reflectance (instead of 480 in the benchmark), these errors are quite small, implying that the new $\delta$-M+ method performs satisfactorily.

Figure 10 shows the relative errors of the Kokhanovsky et al. (2010) cloud benchmark results. Once again, the new $\delta-M+$ method greatly reduces the relative error and provides satisfactory results.

Finally, a slab of particles with the extremely forwardpeaked FF scattering phase function shown in Fig. 7 is used to test the radiance output. The upward radiances at three azimuth angles are shown in Fig. 11, where the incident beam angle is $30^{\circ}$ and the slab optical thickness is set to be 0.5 . The results show strong oscillations of the radiances computed with the original $\delta$ - $M$, which could not yield converged results even for 200 streams, while the new $\delta-M+$ method provides smooth and stable results for only 32 streams.

\section{Summary and discussion}

The $\delta$ - $M+$ method is an enhanced version of the $\delta-M$ method that maintains the same computational efficiency but improves the accuracy significantly. By applying Gaussian weights $w_{\ell} f$ to represent the truncation peak, the new $\delta-M+$ method eliminates the errors of the Legendre moments beyond the first $M$ expansion terms and produces accurate radiances for practical applications involving strongly forward-peaked scattering phase functions associated with large aerosol, cloud particle, and coastal (turbid) water particles.
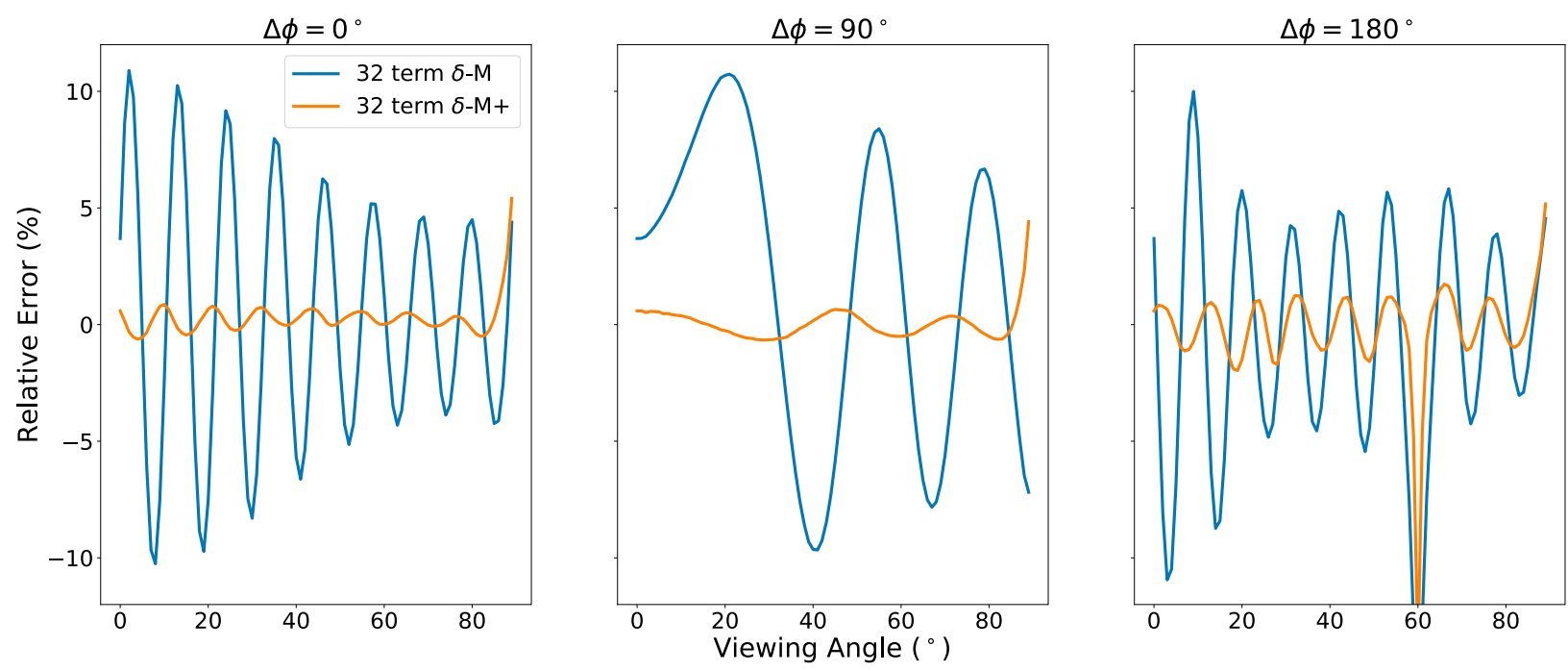

FIG. 9. Relative error (\%) for the Kokhanovsky et al. (2010) aerosol benchmark Results at three relative azimuths are shown: (left) $0^{\circ}$, (center) $90^{\circ}$, and (right) $180^{\circ}$. 

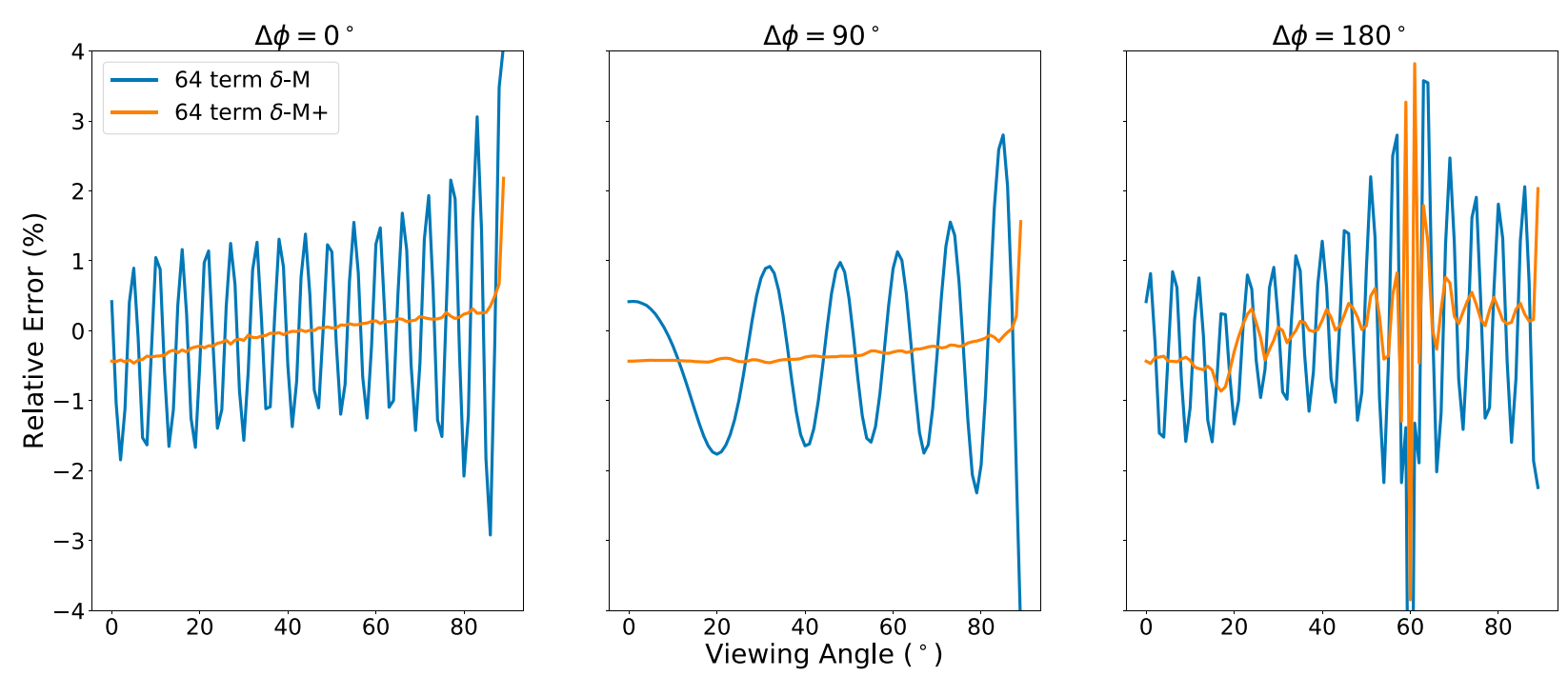

FIG. 10. As in Fig. 9, but for the cloud benchmark.

By accurately representing a majority of the Legendre moments, the $\delta-M+$ algorithm is mathematically elegant, accurate, reliable, and computationally fast. As in the original $\delta$ - $M$ method, the truncation factor of the forward peak is automatically determined by $w_{0} f$. The combination of the $\delta$-M method with the singlescattering correction can also be easily accomplished. These advantages make the new $\delta$ - $M+$ method a practical tool that is simple and easy to implement in most radiative transfer models.
The $\delta$ - $M+$ algorithm has been implemented in the latest DISORT model available (at http://lllab.phy. stevens.edu/disort/; Lin et al. 2015) as well as the AccuRT radiative transfer model (Hamre et al. 2017; K. Stamnes et al. 2017).

Future work, aimed at improvement and extension of the new $\delta-M+$ method, includes 1 ) finding a possibly better weighting function to replace the simple Gaussian function, 2) testing the performance of the $\delta$ - $M+$ method when combined with the single-scattering correction and
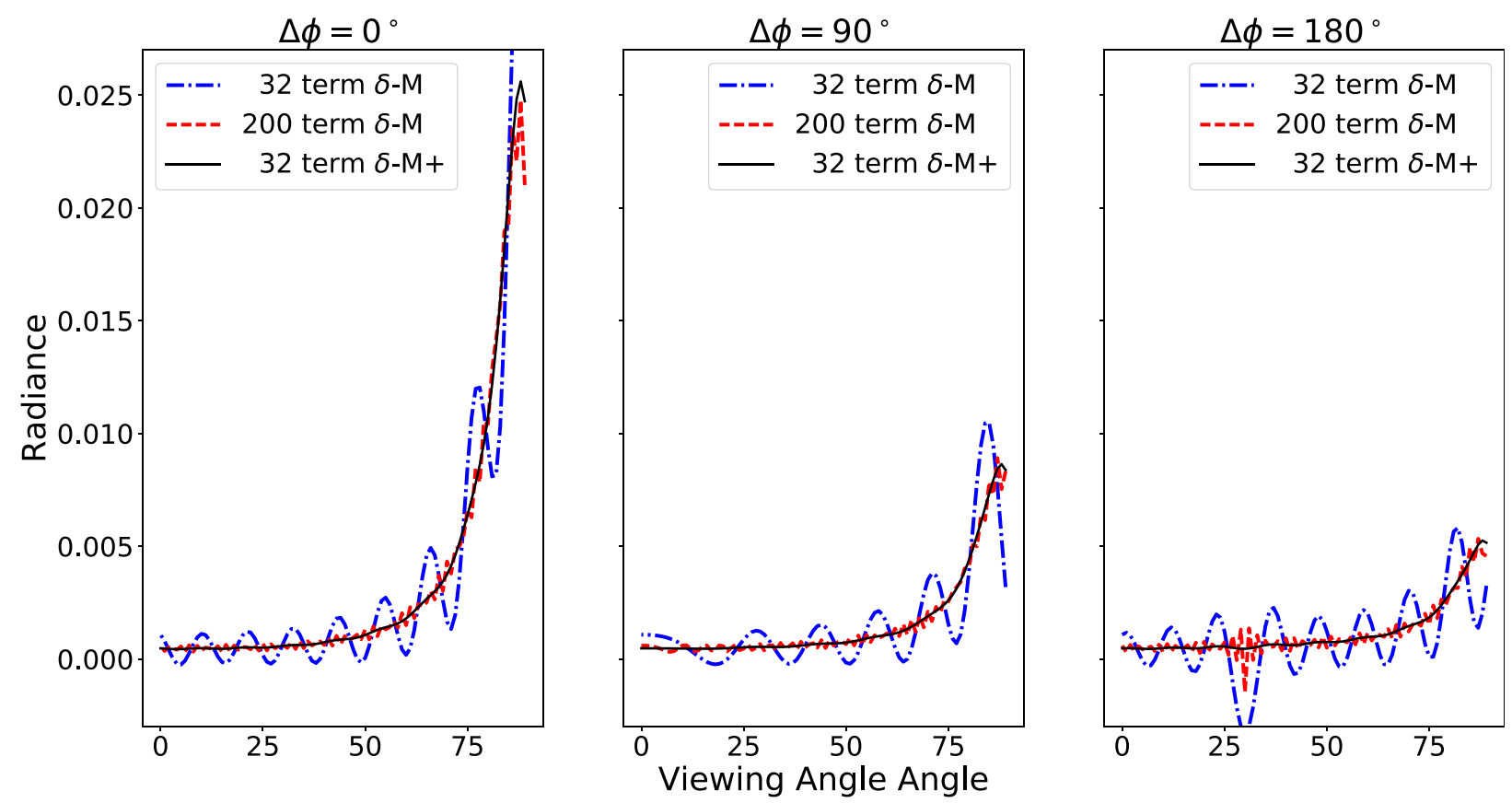

FIG. 11. Upward radiance from a homogeneous layer with an FF scattering phase function. Results at three relative azimuths are shown: (left) $0^{\circ}$, (center) $90^{\circ}$, and (right) $180^{\circ}$. 
comparing it to the $\delta$-fit and other truncation methods, and 3) applying the $\delta-M+$ method to represent the phase matrix in vector (polarized) radiative transfer models (Min and Duan 2004; He et al. 2007; Zhai et al. 2009; Cohen et al. 2013; S. Stamnes et al. 2017).

Acknowledgments. This work was partially funded by the NASA Aerosols-Clouds-Ecosystems (ACE) mission.

\section{REFERENCES}

Arfken, G. B., and H. J. Weber, 1995: Mathematical Methods for Physicists. 4th ed. Academic Press, 1029 pp.

Berk, A., P. Conforti, R. Kennett, T. Perkins, F. Hawes, and J. van den Bosch, 2014: MODTRAN6: A major upgrade of the MODTRAN radiative transfer code. Algorithms and Technologies for Multispectral, Hyperspectral, and Ultraspectral Imagery $X X$, M. Velez-Reyes and F. A. Kruse, Eds., Society of Photo-Optical Instrumentation Engineers (SPIE Proceedings, Vol. 9088), 90080H, https://doi.org/10.1117/12.2050433.

Cohen, D., S. Stamnes, T. Tanikawa, E. Sommersten, J. Stamnes, J. Lotsberg, and K. Stamnes, 2013: Comparison of discrete ordinate and Monte Carlo simulations of polarized radiative transfer in two coupled slabs with different refractive indices. Opt. Express, 21, 9592-9614, https://doi.org/10.1364/OE.21.009592.

Fournier, G. R., and J. L. Forand, 1994: Analytic phase function for ocean water. Ocean Optics XII, J. S. Jaffe, Ed., Society of Photo-Optical Instrumentation Engineers (SPIE Proceedings, Vol. 2258), 194-201, https://doi.org/10.1117/12.190063.

Hamre, B., S. Stamnes, K. Stamnes, and J. Stamnes, 2017: AccuRT: A versatile tool for radiative transfer simulations in the coupled atmosphere-ocean system. Proc. AIP Conf. on Radiation Processes in the Atmosphere and Ocean, Auckland, New Zealand, American Institute of Physics, 120002, https://doi.org/ 10.1063/1.4975576

He, X., D. Pan, Y. Bai, Q. Zhu, and F. Gong, 2007: Vector radiative transfer numerical model of coupled ocean-atmosphere system using matrix-operator method. Sci. China, 50D, 442-452, https://doi.org/10.1007/s11430-007-2075-4.

Henyey, L. G., and J. L. Greenstein, 1941: Diffuse radiation in the galaxy. Astrophys. J., 93, 70-83, https://doi.org/10.1086/144246.

Hu, Y.-X., B. Wielicki, B. Lin, G. Gibson, S.-C. Tsay, K. Stamnes, and T. Wong, 2000: $\delta$-fit: A fast and accurate treatment of particle scattering phase functions with weighted singular-value decomposition least-squares fitting. J. Quant. Spectrosc. Radiat. Transfer, 65, 681690, https://doi.org/10.1016/S0022-4073(99)00147-8.

Jin, Z., T. P. Charlock, K. Rutledge, K. Stamnes, and Y. Wang, 2006: Analytical solution of radiative transfer in the coupled atmosphere-ocean system with a rough surface. Appl. Opt., 45, 7443-7455, https://doi.org/10.1364/AO.45.007443.

Joseph, J. H., W. Wiscombe, and J. Weinman, 1976: The deltaEddington approximation for radiative flux transfer. J. Atmos. Sci., 33, 2452-2459, https://doi.org/10.1175/1520-0469(1976)033<2452: TDEAFR $>2.0 . \mathrm{CO} ; 2$.

Kokhanovsky, A. A., and Coauthors, 2010: Benchmark results in vector atmospheric radiative transfer. J. Quant. Spectrosc. Radiat. Transfer, 111, 1931-1946, https://doi.org/10.1016/ j.jqsrt.2010.03.005.

Lin, Z., S. Stamnes, Z. Jin, I. Laszlo, S.-C. Tsay, W. Wiscombe, and K. Stamnes, 2015: Improved discrete ordinate solutions in the presence of an anisotropically reflecting lower boundary: Upgrades of the DISORT computational tool. J. Quant. Spectrosc. Radiat. Transfer, 157, 119-134, https://doi.org/ 10.1016/j.jqsrt.2015.02.014.

Min, Q., and M. Duan, 2004: A successive order of scattering model for solving vector radiative transfer in the atmosphere. J. Quant. Spectrosc. Radiat. Transfer, 87, 243-259, https://doi.org/10.1016/ j.jqsrt.2003.12.019.

Mitrescu, C., and G. Stephens, 2004: On similarity and scaling of the radiative transfer equation. J. Quant. Spectrosc. Radiat. Transfer, 86, 387-394, https://doi.org/10.1016/j.jqsrt.2003.12.028.

Nakajima, T., and M. Tanaka, 1988: Algorithms for radiative intensity calculations in moderately thick atmospheres using a truncation approximation. J. Quant. Spectrosc. Radiat. Transfer, 40, 51-69, https://doi.org/10.1016/0022-4073(88)90031-3.

Rozanov, V. V., and A. I. Lyapustin, 2010: Similarity of radiative transfer equation: Error analysis of phase function truncation techniques. J. Quant. Spectrosc. Radiat. Transfer, 111, 1964 1979, https://doi.org/10.1016/j.jqsrt.2010.03.018.

_ - A. Rozanov, A. Kokhanovsky, and J. Burrows, 2014: Radiative transfer through terrestrial atmosphere and ocean: Software package SCIATRAN. J. Quant. Spectrosc. Radiat. Transfer, 133, 13-71, https://doi.org/10.1016/j.jqsrt.2013.07.004.

Spurr, R., 2008: LIDORT and VLIDORT: Linearized pseudospherical scalar and vector discrete ordinate radiative transfer models for use in remote sensing retrieval problems. Light Scattering Reviews 3: Light Scattering and Reflection, Springer, 229-275.

Stamnes, K., and J. J. Stamnes, 2016: Radiative Transfer in Coupled Environmental Systems: An Introduction to Forward and Inverse Modeling. Wiley, $368 \mathrm{pp}$.

_ cally stable algorithm for discrete-ordinate-method radiative transfer in multiple scattering and emitting layered media. Appl. Opt., 27, 2502-2509, https://doi.org/10.1364/AO.27.002502.

$\longrightarrow,-\longrightarrow, \ldots$, and I. Laszlo, 2000: DISORT, a general-purpose Fortran program for discrete-ordinate-method radiative transfer in scattering and emitting layered media: Documentation of methodology. Stevens Institute of Technology Dept. of Physics and Engineering Physics Tech. Rep., 107 pp.

_ - G. E. Thomas, and J. J. Stamnes, 2017: Radiative Transfer in the Atmosphere and Ocean. 2nd ed. Cambridge University Press, $528 \mathrm{pp}$.

Stamnes, S., S. Ou, Z. Lin, Y. Takano, S. Tsay, K. Liou, and K. Stamnes, 2017: Polarized radiative transfer of a cirrus cloud consisting of randomly oriented hexagonal ice crystals: The $3 \times 3$ approximation for non-spherical particles. J. Quant. Spectrosc. Radiat. Transfer, 193, 57-68, https://doi.org/10.1016/ j.jqsrt.2016.07.001.

Takano, Y., and K.-N. Liou, 1989: Solar radiative transfer in cirrus clouds. Part I: Single-scattering and optical properties of hexagonal ice crystals. J. Atmos. Sci., 46, 3-19, https://doi.org/ 10.1175/1520-0469(1989)046<0003:SRTICC $>2.0 . C O ; 2$.

Wiscombe, W., 1977: The delta- $M$ method: Rapid yet accurate radiative flux calculations for strongly asymmetric phase functions. J. Atmos. Sci., 34, 1408-1422, https://doi.org/ 10.1175/1520-0469(1977)034<1408:TDMRYA > 2.0.CO;2.

Zhai, P.-W., Y. Hu, C. R. Trepte, and P. L. Lucker, 2009: A vector radiative transfer model for coupled atmosphere and ocean systems based on successive order of scattering method. $O p t$ Express, 17, 2057-2079, https://doi.org/10.1364/OE.17.002057. 